Referencia para citar este artículo: Pavez-Soto, I., León-Valdebenito, C. \& Triadú-Figueras, V. (2016). Jóvenes universitarios y medio ambiente en Chile: percepciones y comportamientos. Revista Latinoamericana de Ciencias Sociales, Niñez y Juventud, 14 (2), pp. 1435-1449.

\title{
Jóvenes universitarios y medio ambiente en Chile: Percepciones y comportamientos*
}

\author{
ISKRA PAVEZ-SOTO** \\ Profesora Universidad Bernardo O’Higgins y Universidad del Desarrollo, Chile. \\ CAROLINA LEÓN-VALDEBENITO *** \\ Profesora Universidad Bernardo O’Higgins, Chile. \\ VERÓNICA TRIADÚ-FIGUERAS **** \\ Socióloga Universidad Bernardo O'Higgins, Chile.
}

\section{Artículo recibido en diciembre 4 de 2015; artículo aceptado en marzo 29 de 2016 (Eds.)}

- Resumen (descriptivo): La preocupación por el medio ambiente en Chile aumentó durante los últimos años entre las y los jóvenes universitarios, un actor social clave con capacidad de influir en el debate público. En este artículo analizamos los resultados de una encuesta realizada a jóvenes universitarios de Santiago sobre sus percepciones, actitudes, conocimientos y comportamientos proambientales. Se concluye que el $76 \%$ de las y los jóvenes universitarios tiene una negativa percepción del medio ambiente urbano debido a la contaminación atmosférica. También que los espacios públicos están contaminados y degradados en los sectores más excluidos. Aunque existe una actitud de preocupación, una demanda por educación ambiental y un alto conocimiento de temas ambientales, solo un 23\% de jóvenes universitarios ha realizado comportamientos proambientales como actividades de incidencia politica, reciclaje y sensibilización.

Palabras clave: Jóvenes universitarios, medio ambiente natural, percepción (Tesauro de Ciencias Sociales de la Unesco).

\section{University students and the environment in Chile: Perception and behaviour}

- Abstract (descriptive): Concern about the environment in Chile has increased during recent years among university students, a key social actor that has the ability to influence public debate. This survey analyses the perceptions, attitudes, knowledge and pro-environmental behaviour of a group of university students from Santiago. We concluded that $76 \%$ of the university students that participated in the study have a negative perception of urban environment due to air pollution. Also we found that the public spaces have been polluted and degraded around socially excluded areas. In spite of attitudes concern, environmental education demands and strong environmental knowledge, only

\footnotetext{
* Este artículo corto hace parte del proyecto denominado "Jóvenes Universitarios y Medio Ambiente en el Chile del siglo XXI" financiado por la Dirección de Investigación de la Universidad Bernardo O'Higgins con el código DIRI No 0001/2014, realizado entre el 1 de junio 2014 al 30 de junio 2015. La investigación fue descriptiva cuantitativa. Para correspondencia: iskra.pavez@ubo.cl Área de conocimiento: Ciencias Sociales; Subárea: Sociología medioambiental. Las autoras expresan su agradecimiento a Eduardo Herrera Vicencio, Víctor Peña Vásquez, Osvaldo Vejar Fuentealba y Juan Faúndez Alvarado.

** Universidad Bernardo O’Higgins, Observatorio Regional de Paz y Seguridad (Orpas) y Universidad del Desarrollo, Facultad de Gobierno. Correos electrónicos: iskra.pavez@ubo.cl; i.pavez@udd.cl

*** Universidad Bernardo O’Higgins, Centro de Investigación en Recursos Naturales y Sustentabilidad (Cirenys). Correo electrónico: carolina.leon@ubo.cl

**** Universidad Bernardo O’Higgins, Observatorio Regional de Paz y Seguridad (Orpas). Correo electrónico: vtriadu@gmail.com
} 
23\% of university students have developed pro-environmental behaviour such as political advocacy, recycling or awareness activities.

Key words: Young, environment, perception (Unesco Social Sciences Thesaurus).

\section{Jovens universitários e meio ambiente do Chile: percepções e comportamentos}

- Resumo (descritivo): A preocupação pelo meio ambiente no Chile cresceu durante os últimos anos entre os/as jovens universitários/as, que são atores sociais chave com capacidade de influenciar o debate público. Neste artigo, são analisados os resultados de uma pesquisa realizada com jovens universitários da cidade de Santiago sobre as suas percepções, atitudes, conhecimentos e comportamentos pró-ambientais. Conclui-se que 76\% de os/as jovens universitários/as têm uma percepção negativa do meio ambiente urbano, devido à alta poluição do ar e por os espaços públicos estarem contaminados e degradados nos setores mais excluidos. Identifica-se que, apesar de haver uma atitude de preocupação, de demanda por uma educação ambiental e de um alto conhecimento de temas ambientais, somente 23\% dos/das jovens universitários/as desenvolvem comportamentos pró-ambientais como atividades de incidência política, reciclagem e sensibilização.

Palavras-chave: Jovens, meio ambiente, percepção (Thesaurus de Ciências Sociais da Unesco).

-1. Introducción. -2. Método. -3. Marco conceptual. -4. Resultados y discusión. -4.1. Percepción del medio ambiente. -4.2. Actitudes hacia el medio ambiente. -4.3. Conocimiento ambiental. -4.4. Comportamiento proambiental. -5. Conclusiones. -Lista de referencias.

\section{Introducción}

El objetivo de este artículo es reflexionar sobre la relación que tienen las y los jóvenes estudiantes universitarios de Santiago de Chile con el medio ambiente. Recientemente, en nuestro país, las y los jóvenes estudiantes universitarios han sido protagonistas de varios movimientos sociales que han logrado instalar en el debate público temas tan importantes como la educación o la protección del medio ambiente (especialmente frente a determinados proyectos energéticos), concentrándose un número importante de estas manifestaciones en la ciudad de Santiago de Chile.

En el colectivo juvenil, las y los estudiantes universitarios, como subgrupo, están situados en una doble posición de poder. Por un lado, en el presente son un actor social con capacidad de presión e incidencia, ya que a través de su movilización pueden influir en el debate público y generar grandes transformaciones sociales (Castillo, 2007, Camargo, 2009, Báez, 2010, Arias-Cardona \& Alvarado, 2015, Castro \& Saavedra, 2013).

En Chile esto se expresa en los importantes logros del Movimiento Estudiantil - compuesto principalmente por jóvenes- que logró instalar en el debate público el tema de la educación y actualmente se lleva a cabo una reforma educativa (Avendaño, 2014). Por otro lado, en el futuro, una vez que las y los jóvenes universitarios egresen, se titulen y se integren al mercado laboral, ocuparán posiciones de poder, autoridad y liderazgo en las organizaciones y las instituciones en las cuales se desempeñen, serán "tomadores de decisión", por lo tanto, sus acciones y decisiones, eventualmente, impactarán sobre el medio ambiente (ParkerGumucio \& Muñoz-Rau, 2012). Por ello, es de vital importancia conocer sus valores $y$ actitudes en esta materia.

Según una encuesta realizada por la Universidad Andrés Bello (2013), al 60\% de la población chilena adulta le preocupa mucho el medio ambiente y desarrollan actitudes de protección como reciclar (el 66\% lo hace, el resto se justifican por la inexistencia de lugares para realizarlo) y ahorrar energía eléctrica (el $85 \%$ apaga las luces cuando no se precisa que estén encendidas); la mayoría (85\%) asocia su conservación con calidad de vida y ahorro económico. Por otro lado, para el $37 \%$ de la población, la contaminación del aire es el principal problema ambiental y lo atribuyen a las industrias (46\%) y al transporte (31\%). 
Según datos del Ministerio del Medio Ambiente (2014), el $50 \%$ de la población chilena tendría conductas de protección ambiental, especialmente las mujeres y quienes residen en la ciudad de Santiago.

Parker-Gumucio y Muñoz-Rau (2012, p. 205) registraron que las y los jóvenes universitarios estudiantes de carreras denominadas de élite (como ingenierías, derecho o medicina) tienen poca información sobre el medio ambiente, debido a la falta de educación ambiental en los programas curriculares. Si bien desde los años noventa algunas universidades chilenas han implementado cursos optativos sobre la materia, según Muñoz-Pedreros (2014) esto no ha sido incorporado como un contenido transversal en el currículum de todas las carreras universitarias. Por su parte, Heyl, MoyanoDíaz y Cifuentes (2013) constataron que estudiantes de ingeniería de una universidad chilena tendrían actitudes altamente positivas hacia el reciclaje, la eficiencia energética y el uso responsable del agua y en menor medida hacia el consumo responsable de productos manufacturados, la participación en actividades de protección ambiental y los medios de transporte sustentables; en términos de sexo, las mujeres tendrían más comportamientos proambientales que los hombres. Además, en la $7^{a}$ Encuesta Nacional de Juventud (Injuv, 2013, p. 103), se registró que un 54\% está interesado en el cuidado del medio ambiente, demostrando valores modernos que forman parte de la "nueva política" -junto a los derechos de las mujeres y de las minorías sexuales- (Dalton, 2006, citado en Injuv, 2013 p. 118).

Por último, se encuentran otros estudios sobre medio ambiente y adolescentes, tal como Barazarte-Castro, Neaman, Vallejo-Reyes y García-Elizalde (2014) sobre los colegios certificados por el Sistema Nacional de Certificación Ambiental de Establecimientos Educacionales (Sncae)-un programa coordinado por el Ministerio del Medio Ambiente y el Ministerio de Educación-; Aguayo (2005) sobre el conocimiento ambiental de estudiantes de la Enseñanza Básica en colegios con y sin certificación medioambiental; y MoyanoDíaz, Encina y Vicente (2007), en torno a la actitud y el comportamiento proambiental de estudiantes de la Enseñanza Media. A la luz de estos antecedentes se evidencia que la relación de las y los jóvenes universitarios con el medio ambiente ha sido abordado de forma incipiente en Chile, por ello este artículo aporta un primer diagnóstico sobre sus percepciones, actitudes y conocimientos, a fin de fortalecer el comportamiento proambiental en este actor social clave que serán los futuros "tomadores de decisiones".

\section{Método}

Para recopilar los datos se diseñó una encuesta que contenía preguntas cerradas, otras con opciones tipo Escala de Likerty algunas abiertas; para su elaboración se tomaron como referencia y adaptaron algunas preguntas de las encuestas de Barazarte-Castro et al. (2014), OliverTrobat, Castells-Valdivielso, Casero-Martínez y Morey-López, (2005) y de la Universidad Andrés Bello (2013); se analizaron cuatro variables: percepción; actitudes, conocimiento y comportamiento proambiental, considerando las diferencias de sexo, el nivel socioeconómico familiar, la carrera y el tipo de Universidad. Con un intervalo de confianza del $90 \%$, la muestra aleatoria estuvo constituida por 119 jóvenes con las siguientes características sociodemográficas: 66 mujeres y 53 hombres; de 18 a 24 años de edad; de diversas carreras profesionales, aunque con mayorpreponderancia de las ingenierías, ${ }^{1}$ provenientes de diversos niveles socioeconómicos: muy alto $(8,40 \%)$, alto $(28,57 \%)$, medio $(31,93 \%)$, medio-bajo $(28,57 \%)$ y bajo $(2,52 \%),{ }^{2} 58$ estudiantes pertenecientes a Universidades del Consejo de Rectores de Chile (Cruch), ${ }^{3}$ caracterizadas como de élite, porque allí estudian principalmente sectores económicamente medios y altos y

1 Las y los estudiantes se distribuían en las siguientes carreras: Agronomía (2), Arquitectura (1), Ciencias (7), Ciencias Políticas (5), Contabilidad y Administración (7), Derecho (2), Diseño y Marketing (5), Educación (19), Ingenierías (63), Literatura (1), Relaciones Públicas (1), Salud (5) y Trabajo Social (1).

2 Niveles socioeconómicos basados en las comunas de residencia declaradas por las y los estudiantes, según Índice de Desarrollo Humano (IDH) comunal de Chile, del Programa de las Naciones Unidas para el Desarrollo (Pnud, 2000, citado en De Mattos, 2002).

3 Universidades pertenecientes al Consejo de Rectores de las Universidades Chilenas (Cruch), tales como PUC (19), U. de Chile (18), Usach (20) y Utem (2). 
61 de Universidades no pertenecientes al Cruch, ${ }^{4}$ orientadas hacia las capas medias y bajas de la sociedad chilena, quienes acceden principalmente a través de créditos y becas (Parker-Gumucio \& Muñoz-Rau, 2012). La tabulación de los datos se realizó empleando el programa estadístico SPSS y la prueba del Chicuadrado de Pearson.

\section{Marco conceptual}

En las últimas décadas los problemas ambientales han ido cobrando mayor relevancia para la sociedad. Elementos forzantes como el cambio de uso de suelo por actividad humana, el cambio climático y la contaminación, hacen proyectar impactos significativamente negativos sobre la biodiversidad y los servicios que los ecosistemas entregan a la humanidad (Sala, Chapin, Armesto, Berlow, Bloomfield, Dirzo, 2000). Una de las respuestas a esta crisis ambiental ha sido la sensibilización de la ciudadanía a fin de convivir con el entorno, preservarlo, y hacer un uso sustentable del mismo para transformar las percepciones que se tienen de él (Novo, 2009). Siguiendo a Alea (2006, p. 9) conceptualizamos la percepción como la comprensión de los diferentes elementos que conforman el medio en el cual nos desenvolvemos y cómo esos condicionantes influyen en nuestro desempeño diario. La percepción que tenemos del medio ambiente influirá considerablemente en la orientación y regulación de nuestras acciones hacia el mismo. Además de la percepción, las actitudes entregan información sobre la relación de las personas con el medio ambiente, entendido este en su dimensión local (entorno urbano natural) y global (la naturaleza en general) (Ministerio del Medio Ambiente, 2010). Las actitudes se conceptualizan como los sentimientos favorables o desfavorables que se tienen hacia alguna característica del medio o hacia un problema relacionado con él (Holahan, 2006). Según Le Breton (1999, 2013), las actitudes pueden ser vistas como "sentimientos"; porque las formas de expresar y nombrar las emociones están determinadas por el repertorio común

4 Universidades tales como U. Autónoma (1), U. Central (6), UBO (11), Udla (12), UDP (3), Unab (17) y UST (10). de un grupo social. Conocer las actitudes y los sentimientos ayudará a predecir las conductas proambientales. Aun cuando se debe tener en consideración que las correlaciones entre actitudes proambientales y conductas ecológicamente responsables en general no son altas (Álvarez \& Vega, 2009).

Numerosos autores (Alea, 2006, Duerden \& Witt, 2010, Félonneau \& Becker, 2008, Hess \& Waló, 2001, Stern, 2000, Taylor \& Todd, 1995) señalan que a mayor nivel de conocimiento ambiental formal posean los individuos, habría una mayor tendencia hacia actitudes proambientales. Meinhold y Malkus (2005) señalan que estudiantes que han tenido cursos especializados en medio ambiente desarrollan mayores actitudes proambientales en comparación con quienes no los han tenido. Conclusiones similares se han encontrado en Rojas (2009).

Considerando la percepción, las actitudes y los conocimientos es posible analizar de forma general la relación de los individuos con el medio ambiente $\mathrm{y}$, a partir de estos elementos, hacer una proyección del comportamiento proambiental que tendrán y cómo fomentarlo. El comportamiento proambiental es definido por Gómez-Benito et al. (1999, citado en Sevillano \& Aragonés, 2009, p. 133) como la preocupación personal por el medio ambiente, la acción personal proambiental y la participación en acciones colectivas proambientales, es decir, la coherencia entre la actitud y la conducta del individuo. Por su parte, Kaiser, Wölfing y Fuhrer (1999, p. 1, citado en Heyl et al., 2013, p. 488) definen el comportamiento proambiental como las acciones que contribuyen en la preservación y conservación del medio ambiente.

\section{Resultados y discusión}

Los datos muestran que existe interés y preocupación entre las y los jóvenes universitarios por las condiciones del medio ambiente urbano local de la ciudad de Santiago $\mathrm{y}$ por la protección de la naturaleza en general. En nuestro estudio hallamos que las percepciones, las actitudes y los conocimientos inciden en el comportamiento proambiental de las y los jóvenes universitarios en dos niveles: a 
nivel microsocial, en los valores y las prácticas que llevan a cabo en la vida cotidiana respecto al medio ambiente; y a nivel macrosocial, en tanto conforman un actor social con capacidad de incidencia pública en este tema.

\subsection{Percepción del medio ambiente}

En concordancia con las encuestas de los organismos públicos anteriormente comentadas, en el Gráfico 1, letra $\mathrm{A}$, podemos observar que las y los jóvenes encuestados perciben que las condiciones del medio ambiente en Santiago son malas. Desde el punto de vista socioeconómico, las y los jóvenes que proceden de comunas con nivel socioeconómico o Índice de Desarrollo Humano (IDH) muy alto (40\%) las ven más negativamente, seguidos del grupo alto $(26,5 \%)$ y del medio bajo (26,5\%). Además, las jóvenes del área de Ciencias opinaron en mayor proporción $(30 \%)$ que son muy malas. Según Heyl et al. (2013) existen diferencias significativas entre mujeres y hombres respecto a las percepciones ambientales, pero en nuestra encuesta solo se halló entre estudiantes que cursan el primer año de su carrera profesional. Desde el punto de vista del tipo de centro, un 34\% de estudiantes que pertenecen a universidades del Cruch consideró las condiciones del medio ambiente de Santiago muy malas, mientras que este porcentaje bajó a $20 \%$ entre quienes no forman parte del Cruch, solamente consideraron las condiciones ambientales muy buenas un $2 \%$ de estudiantes exclusivamente pertenecientes a centros Cruch.

Gráfico 1. Percepción de las y los jóvenes universitarios sobre el medio ambiente de Santiago.

\section{Condición del} Medio Ambiente
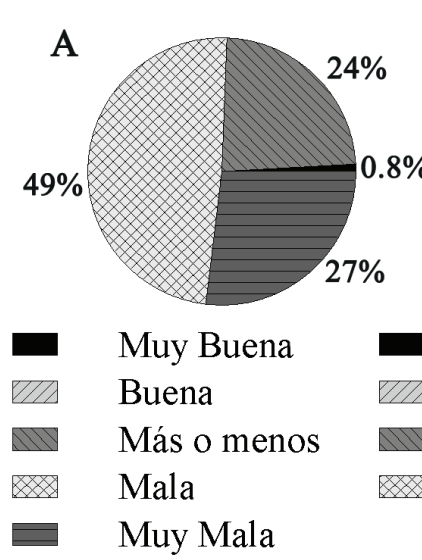

Muy Buena

Buena

Más o menos

Mala

Muy Mala

\section{Importancia del}

Medio Ambiente

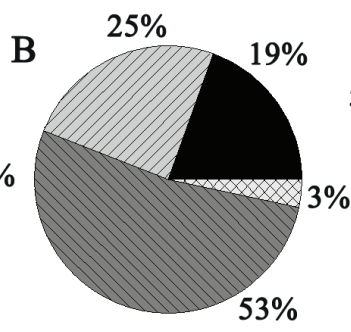

Muy de acuerdo

De acuerdo

Ambos

En desacuerdo

Fuente: Elaboración propia.

También en el Gráfico 1, letra B, observamos que el $52 \%$ de jóvenes cree que el medio ambiente es igual de importante que el desarrollo económico, el $43 \%$ lo considera más importante (sumando muy de acuerdo y de acuerdo), solamente un 3\% discrepa. No se observan diferencias significativas por sexo, nivel socioeconómico ni tipo de centro universitario. También en el Gráfico 1, letra C, se comprueba que el $50 \%$ de las y los encuestados percibe en estado regular (limpios, pero con poco equipamiento y sin áreas verdes) a los espacios

Rev.latinoam.cienc.soc.niñez juv 14 (2): 1435-1449, 2016

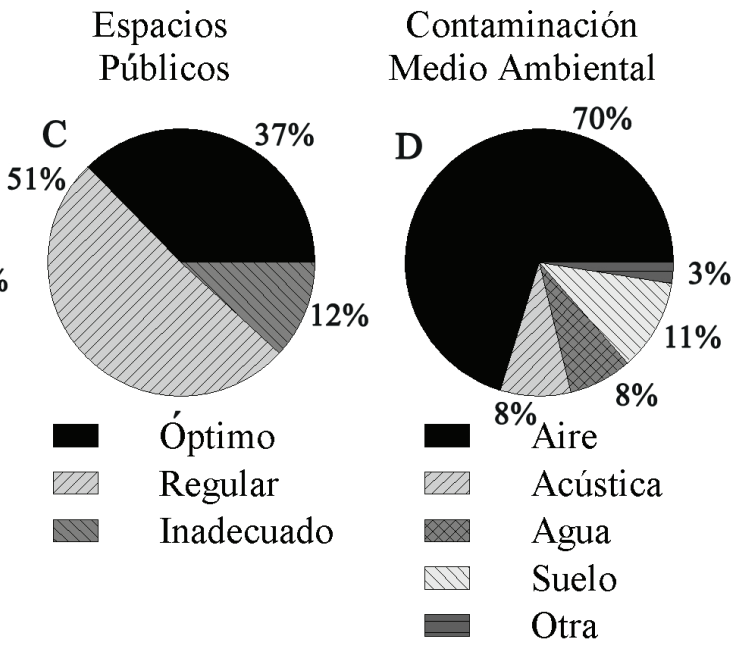

públicos urbanos - como plazas y parquesque usan de forma cotidiana para compartir con sus amistades o familias, el 37\%, óptimos (muy limpios, bien equipados y con áreas verdes); y un $12 \%$, inadecuados (descuidados o contaminados). En las respuestas de las y los estudiantes no se observan diferencias de sexo, pero dentro del grupo de las y los estudiantes que señalan disponer de espacios públicos óptimos, la mayoría proviene de Universidades del Cruch; mientras que la mayoría de quienes habitan espacios públicos inadecuados estudia 
en Universidades que no pertenecen al Cruch. Desde el punto de vista socioeconómico, la mayoría de estudiantes de comunas con un alto Índice de Desarrollo Humano (IDH) los percibe óptimos, quienes habitan en comunas con un IDH medio y medio-bajo, regulares; si bien el estado inadecuado es transversal a todos los niveles socioeconómicos, no aparece en el nivel más alto.

Según Gehl (2006, citado en Valenzuela et al., 2010, p. 89), los espacios urbanos colectivos de uso público -como parques y plazas- permiten la realización de actividades exteriores como caminar, descansar, jugar, observar y conversar, es decir, facilitan la socialización, el encuentro y la integración entre diferentes grupos sociales (como la infancia, la juventud y las personas mayores), la recreación, el contacto con la naturaleza y el deporte. Por lo tanto, su presencia o ausencia tiene un gran impacto en la calidad de vida de las personas, del barrio y de una ciudad.

Actualmente en Chile se da un consenso sobre la escasez de espacios públicos urbanos (como parques y plazas) dentro del área metropolitana de Santiago, especialmente en los sectores más pobres o altamente excluidos, es decir, en comunas con un Índice de Desarrollo Humano más bajo (Reyes \& Figueroa, 2010). Esto ha sucedido, entre otras razones, por la falta de una política integral de desarrollo urbano que considere y articule las dimensiones arquitectónicas, económicas, sociales $\mathrm{y}$ ambientales en la creación de viviendas y barrios. De acuerdo a Reyes y Figueroa (2010, p. 90), además de esta función social, las áreas verdes cumplen un rol importante en la conservación del medio ambiente local, especialmente en cuanto a la renovación de las partículas del aire. Este último aspecto es de vital importancia, porque, en el Gráfico 1, letra $\mathrm{D}$, vemos que el $70 \%$ de las y los jóvenes universitarios encuestados consideran la contaminación del aire como la más importante de Santiago, seguida de la contaminación del suelo, la acústica y la del agua. Recordemos que según datos del Ministerio del Medio Ambiente (2014, p. 15), el 37\% de la población chilena considera como el principal problema medio ambiental a la contaminación del aire de Santiago.

Las y los jóvenes señalan como principales fuentes contaminantes del aire, en orden de importancia, a las industrias y las fábricas, los vehículos motorizados (automóviles, buses del transporte público, etc.), los sistemas de calefacción doméstica a leñay, en menor medida, la acción de las personas o la ciudadanía (solo hubo una mención a las personas fumadoras), $\mathrm{y}$ en último lugar, se nombra la quema de basura. Sin embargo, según las mediciones técnicas del Ministerio del Medio Ambiente (2015), las principales fuentes contaminantes del aire de Santiago son-jerárquicamente- las residenciales $(31 \%)$, el transporte $(31 \%)$, las industrias $(26 \%)$, las evaporativas $(26 \%)$ y las agrícolas $(5 \%) .{ }^{5}$ En el área metropolitana de la ciudad de Santiago, la leña y los areales (quema agrícola) producen la mayor contaminación de material particulado (MP 2,5); mientras que las fuentes móviles, como los vehículos motorizados y las centrales termoeléctricas aportan mayor óxido de nitrógeno (NOx) y las fundiciones de cobre, más óxido de azufre (SOx) (Ministerio del Medio Ambiente, 2011, p. 62). Es decir, la percepción que tienen las y los encuestados sobre las principales fuentes contaminantes del aire coincide con las mediciones técnicas, pero difiere en su nivel de importancia, lo cual deja entrever la necesidad de educación ambiental que permita hacer efectivas las medidas y los planes de descontaminación, atacando las fuentes emisoras claves, como la utilización de leña para la calefacción residencial.

Seguidamente, las y los jóvenes universitarios encuestados consideraron a la contaminación del suelo como la segunda más importante en la ciudad de Santiago, después del aire y la atribuyen a la acción de las propias personas (por ejemplo, botar la basura en el suelo), a la falta de equipamiento urbano (basureros), al exceso de publicidad (carteles) y a la acumulación de escombros. Por su parte, la

5 Las fuentes son fijas (calefacción), móviles (transporte) y fugitivas (polvo), cada una emite distintos gases contaminantes al aire (Ministerio del Medio Ambiente, 2011, p. 60). 
contaminación del agua es atribuida a la propia composición química del agua potable (exceso de cloro) - esto no es lo que reflejan las cifras del Ministerio del Medio Ambiente (2013)- y a los desechos tóxicos generados por las industrias. Esto evidencia nuevamente un desconocimiento sobre el tema, especialmente en lo que respecta al agua potable. Por último, la contaminación acústica es atribuida al tráfico urbano, las y los jóvenes universitarios encuestados consideran que existe un exceso de vehículos motorizados responsables de causar una sensación de ruido mayor a la tolerable.

En estos datos no se observaron grandes diferencias de sexo ni por tipo de Universidad, pero sí por nivel socioeconómico familiar, la contaminación del aire lo mencionaron más quienes provienen de comunas con un Índice de Desarrollo Humano (IDH) alto y muy alto y la contaminación acústica por estudiantes de comunas con IDH de nivel medio.

\subsection{Actitudes hacia el medio ambiente}

En el Gráfico 2, letra A, se ve que los problemas medioambientales les preocupan mucho a las y los jóvenes escuestados $(94,4 \%$ al sumar de acuerdo y muy de acuerdo). Desde el punto de vista socioeconómico, quienes proceden de comunas con un Índice de Desarrollo Humano alto declaran mostrarse, en mayor número de casos, indiferente respecto a la afirmación presentada y quienes estudian carreras de Ciencias y Arquitectura se muestran muy de acuerdo en mayor proporción; además, las mujeres declaran mayor preocupación que los hombres, ya que estos últimos se han declarado indiferentes en más ocasiones. Respecto al tipo de centro, quienes pertenecen a centros Cruch se mostraron muy preocupados $(36,2 \%)$ o indiferentes $(7 \%)$; mientras que estudiantes de centros que no pertenecen al Cruch fueron más moderados - un $62,3 \%$ de sus respuestas fue de acuerdo, mientras que para estudiantes Cruch, fue un $56,9 \%$ de sus respuestas- .

Gráfico 2. Actitudes de las y los jóvenes universitarios hacia el medio ambiente.

Preocupación por el Medio Ambiente

\section{A}

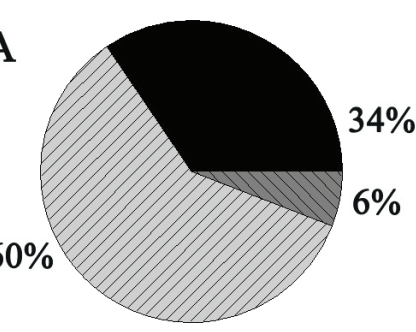

․ㅡㅁ

\begin{abstract}
Muy de acuerdo
\end{abstract}
De acuerdo

Indiferente

En desacuerdo

Muy en desacuerdo
Solución de Problemas Ambientales

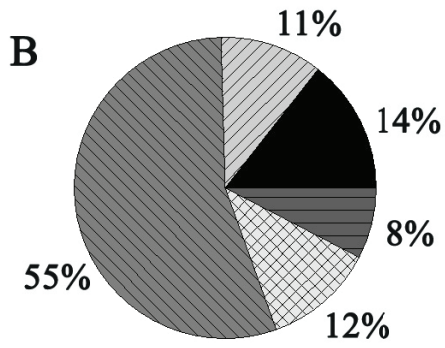

Leyes

Multas

Ed. Ambiental

Incentivos

Otras
Cuidado del

Medio Ambiente

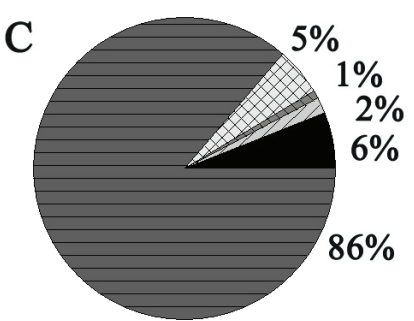

Gobierno

Municipalidad

Empresas

Ciudadanas/os

Todas

Fuente: Elaboración propia.

Así mismo, en el Gráfico 2, letra B, se contata que el $54,6 \%$ de las respuestas considera la educacion ambiental como la principal solución a los problemas ambientales, seguido de leyes más estrictas y multas altas.
Desde el punto de vista de sexo, los hombres prefieren leyes más estrictas y multas altas; mientras las mujeres, educación ambiental. Estas estrategias reflejan los estereotipos de género vigentes en cuanto los hombres optan 
por soluciones más coercitivas y las mujeres por acciones más persuasivas. No hay diferencias significativas según el nivel de IDH, porque la mayoría cree que la educación ambiental es la solución, solo quienes provienen de comunas con IDH muy alto además prefirieron (30\%) los incentivos. Para finalizar, las y los estudiantes que pertenecen a centros Cruch eligieron la educación ambiental en 58,6\% frente al 50,8\% de estudiantes que provienen de centros no Cruch, estos últimos optaron por incentivos para reducir la contaminación $(14,8 \%)$, multas altas $(14,8 \%)$ y leyes más estrictas $(14,8 \%)$, mientras que las y los estudiantes de centros que pertenecen al Cruch optaron por leyes más estrictas $(13,8 \%)$ seguido de otras alternativas $(10,3 \%)$ antes que incentivos y multas, además de educación ambiental.

Por último, en el Gráfico 2, letra C, se registra que el $84,9 \%$ de las y los jóvenes estima que el cuidado del medio ambiente es responsabilidad de todos los actores sociales, aunque quienes cursan carreras de Ciencias consideran más a las empresas y a la ciudadanía y quienes cursan carreras de Ciencias Sociales e Ingeniería, al gobierno y a las municipalidades. Respecto al tipo de centro, quienes no pertenecen al Cruch consideran a la municipalidad como el principal agente $(3,3 \%)$; mientras que las y los estudiantes de centros Cruch, al gobierno $(6,9 \%)$, la ciudadanía $(5,2 \%)$ y las empresas $(1,7 \%)$. Respecto a las diferencias de sexo, los hombres creen que el gobierno $(13,2 \%)$ y las empresas $(1,9 \%)$ deberían proteger al medio ambiente, mientras que las mujeres, en ningún caso destacaron a estos agentes, pero sí a la ciudadanía $(6,1 \%)$ y a la municipalidad $(3 \%)$. Nuevamente, estos últimos datos reflejan los estereotipos de género, porque las mujeres optaron por actores locales, mientras los hombres, por instancias nacionales. La prueba del Chi-cuadrado de Pearson en estos datos corresponde a 0,18 y revela cierta dependencia entre las preferencias y el sexo, lo que permite concluir que las respuestas están condicionadas por los roles de género. Por último, también se observa una alta relación entre las preferencias y el nivel socioeconómico, con un Chi-cuadrado de Pearson correspondiente a 0,004, porque quienes provienen de comunas con IDH alto $\mathrm{y}$ muy alto optaron en mayor proporción por el Gobierno y la ciudadanía.

\subsection{Conocimiento ambiental}

Para conocer el nivel de conocimiento que tenían las y los encuestados, se les preguntó si sabían algo sobre 12 temas relacionados con el medio ambiente. En el Gráfico 3 se observa que las y los estudiantes declaran en un alto porcentaje $(87 \%-68 \%)$ conocer sobre reciclaje, contaminación atmosférica y del agua, cambio climático y deforestación. En un grado intermedio de conocimiento encontramos a la biodiversidad y la eficiencia energética. Los temas menos conocidos son servicios ecosistémicos, desertificación y especies invasoras (16\%-39\%).

Aun cuando un número importante de las y los jóvenes encuestados declara tener conocimientos sobre temáticas ambientales, se evidencia un alto desconocimiento de los nuevos conceptos sobre las amenazas que internacionalmente están siendo destacadas como problemas ambientales claves, por ejemplo las especies invasoras (Chen, 2003) y la desertificación (Reynolds et al., 2007). Temáticas que a nivel nacional también han sido catalogadas como amenazas claves para el medio ambiente (Capp, 2013, Ministerio del Medio Ambiente, 2011, 2013). Por otra parte, se ve una alta desinformación sobre el concepto de servicios ecosistémicos, término que está siendo utilizado para poner en valor los beneficios que entrega la naturaleza a la humanidad (MEA, 2005).

Dentro del grupo de estudiantes que conocían las temáticas se destaca que la principal fuente de información ha sido la enseñanza secundaria, en 11 de los 12 temas, alrededor del $42 \%$ de las y los estudiantes señalan que estas temáticas las aprendieron en el colegio. En segundo lugar (24\%), dentro de las fuentes de información se encuentra la universidad $\mathrm{y}$ en tercer lugar, la televisión (15\%). Estos resultados evidencian un hecho revelado anteriormente: Que las universidades chilenas entregan una incipiente formación ambiental (Parra, 2002, Muñoz-Pedreros, 2014). Un bajo 
número de las y los encuestados señala haber aprendido sobre estos temas al interior de sus familias, donde al parecer existiría poco interés por temas ambientales. Recordemos que según Dalton (2006, citado en Injuv, 2013 p. 118) este interés por el medio ambiente -junto a los derechos de las mujeres y de las minorías sexuales- corresponde a valores modernos que forman parte de la "nueva política". Por tanto, resulta evidente la necesidad de incorporar a las familias como un nuevo actor social clave en los procesos de educación ambiental y desarrollo sustentable (Calvo-Cruz, 2013).

Gráfico 3. Conocimiento de las y los jóvenes universitarios sobre temas ambientales.

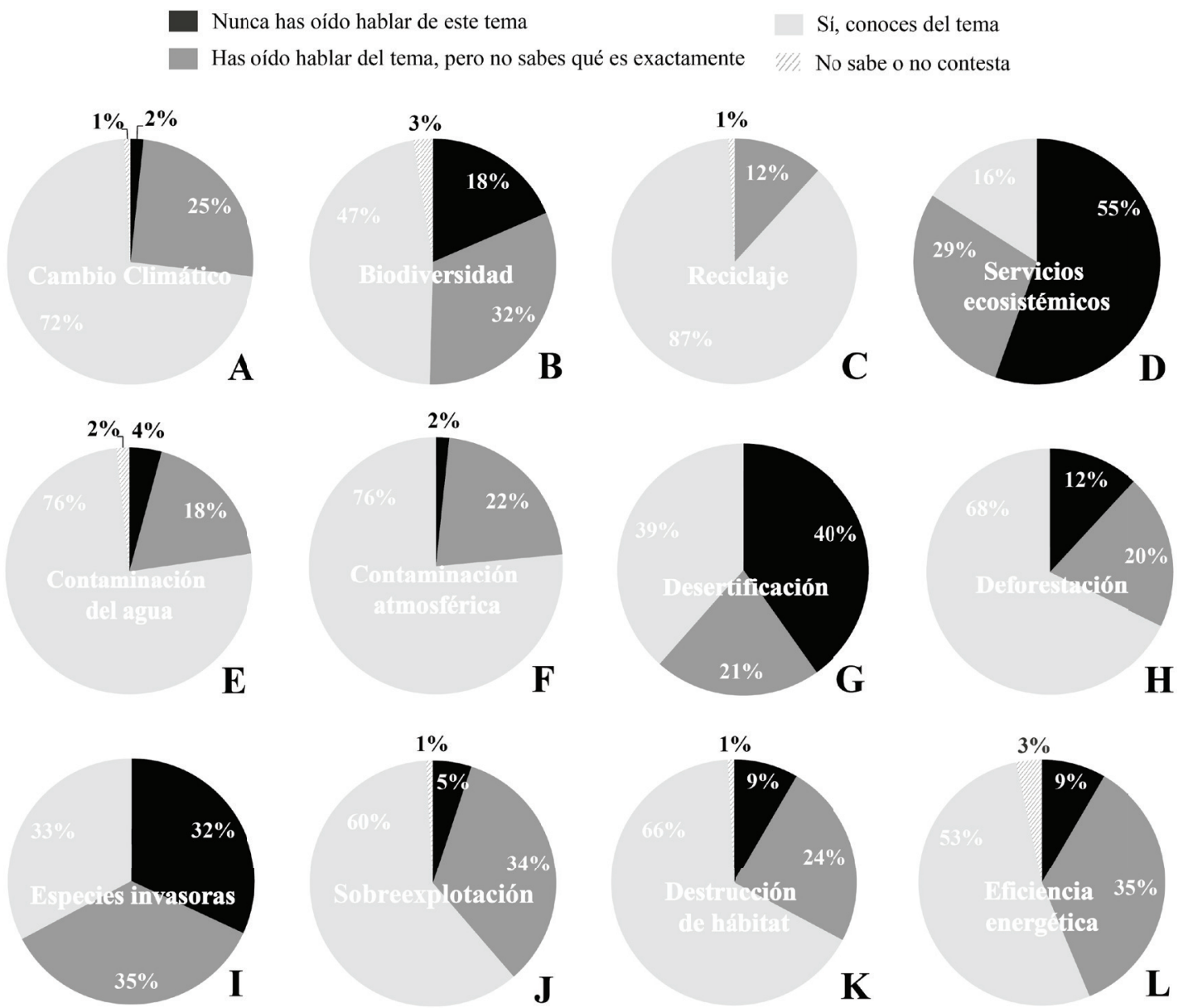

Fuente: Elaboración propia.

\subsection{Comportamiento proambiental}

Para analizar el comportamiento proambiental se preguntó a las y los jóvenes estudiantes universitarios sobre su participación en actividades de protección del medio ambiente en Chile. Los resultados muestran que el $76 \%$ de las y los jóvenes encuestados declara no haber participado en actividades de protección del medio ambiente, mientras que un $23 \%$ sí ha participado. Estos resultados concuerdan con lo señalado por Cerda et al. (2007, citado en Heyl et al., 2013, p. 489) respecto a que la mayoría de las y los estudiantes universitarios chilenos tiene una actitud altamente positiva hacia el medio ambiente, pero una minoría realiza acciones concretas para protegerlo. Por su parte Álvarez y Vega (2009) identifican que la correlación entre actitud proambiental (preocupación por los problemas ambientales) y ejecución 
de conductas ecológicamente responsables es, en general, muy baja, lo que ha llevado a plantear que una alta concientización respecto al medio ambiente, por sí sola, no asegura la puesta en práctica de comportamientos ecológicos responsables, hacen falta planes e incentivos, equipamiento e infraestructura para que se logren llevar a cabo de modo efectivo y permanente.
De las personas que han participado en actividades de protección del medio ambiente, un porcentaje ligeramente mayor corresponde a mujeres, a provenientes de Universidades no pertenecientes al Cruch y del nivel socioeconómico medio. En la Tabla 1 se aprecian, según orden de importancia, las acciones o actividades en las cuales han participado las y los jóvenes encuestados:

Tabla 1. Actividades de protección ambiental en que participan jóvenes universitarios.

\begin{tabular}{|c|c|}
\hline Tipo de actividad & Área o temática específica \\
\hline \multirow{4}{*}{$\begin{array}{c}\text { Marchas callejeras o } \\
\text { cicletadas }\end{array}$} & Rechazo del proyecto hidroeléctrica Hidro Aysen \\
\hline & Rechazo del proyecto hidroeléctrica Alto Maipo \\
\hline & Rechazo del proyecto termoeléctrica Punta de Choros \\
\hline & Otros proyectos hidroeléctricos/termoeléctricos \\
\hline \multirow{3}{*}{ Reciclaje } & Planes comunales municipales de reciclaje \\
\hline & Talleres escolares de reciclaje \\
\hline & Talleres universitarios de reciclaje \\
\hline \multirow{4}{*}{ Defensa de áreas } & Dunas \\
\hline & Isla Juan Fernández \\
\hline & Glaciares \\
\hline & Panul \\
\hline Apoyo trabajo de ONG & Greenpeace Chile \\
\hline \multirow{3}{*}{ Recolección de basuras } & En cerros \\
\hline & Planes comunales de limpieza \\
\hline & Limpieza de playas \\
\hline $\begin{array}{c}\text { Campañas de } \\
\text { sensibilización }\end{array}$ & Reforestación de Patagonia y Parque Nacional Torres del Paine \\
\hline \multirow{2}{*}{ Participación en grupos } & Club escolares \\
\hline & Asociación de Guías y Scouts de Chile \\
\hline Recolección de firmas & No especifica \\
\hline \multirow{4}{*}{ Otras } & Iniciativa gubernamental "Vive tus parques" \\
\hline & Creación de huertos \\
\hline & Talleres de autocultivo \\
\hline & Estilo de vida vegano \\
\hline
\end{tabular}

Fuente: Elaboración propia.

Se observa que las y los jóvenes encuestados participan en actividades consideradas clásicamente como de denuncia o protesta, tales como marchas callejeras o cicletadas masivas (estas últimas han surgido en el último tiempo), cabe señalar que es característico que la mayoría de estas estrategias de manifestación política se han realizado en el entorno urbano de la ciudad de Santiago, especialmente en plazas o avenidas situadas en el epicentro de la ciudad 
como la llamada Plaza Italia o la Alameda, los cuales constituyen lugares emblemáticos de expresión ciudadana. La utilización de las marchas como un mecanismo efectivo para manifestar una visión crítica (por ejemplo, frente a un determinado proyecto energético), probablemente, se deba al gran impacto mediático que tuvieron las marchas realizadas por el Movimiento Estudiantil durante los años 2011 y 2012, consagrándose como un método efectivo de denuncia y opinión (Avendaño, 2014).

Es interesante destacar que estas marchas o cicletadas que se realizan por el centro de la ciudad de Santiago son utilizadas por las y los jóvenes universitarios como un medio para criticar determinados proyectos energéticos de hidroeléctricas o termoeléctricas, los cuales pueden llevarse a cabo en esta misma ciudad o en localidades lejanas. Por cierto, la marcha contra el proyecto energético Hidro Aysén fue la actividad de protección del medio ambiente más mencionada por las y los jóvenes encuestados, es necesario destacar que el mayor impacto de ese proyecto iba a suceder a más de dos mil kilómetros de Santiago, aunque contemplara una línea de transmisión de energía desde la región de Aysén hasta la capital. El hecho de que un proyecto energético sea criticado por la ciudadanía e incluso se organicen marchas en su contra visibiliza un tema poco considerado hasta ahora, lo cual es la participación ciudadana en la elaboración y aprobación de proyectos energéticos que tienen un gran impacto social y ambiental (Leal-Arancibia \& NegronOyarzo, 2012). Las marchas han logrado mostrar que no bastan criterios técnicos y económicos para decidir sobre estas cuestiones, hoy en día resulta indispensable considerar la opinión y participación de toda la comunidad, especialmente de las y los jóvenes universitarios, ya que estos últimos han emergido como un nuevo actor social, generando un gran impacto mediático para incidir y transformar la agenda pública en diversos temas.

En segundo lugar, aparecen las acciones orientadas a promover o ejecutar el reciclaje. El estudio de Heyl et al. (2013, p. 496) señala que la ejecución de algunos comportamientos proambientales, como el reciclaje, está influido por diversos factores, ya sea una actitud positiva hacia el medio ambiente, pero también requiere de una adecuada infraestructura técnica y logística para llevarse a cabo. Recordemos que el propio Ministerio del Medio Ambiente (2014) reconoce que para reciclar no basta la sensibilización, es imprescindible la existencia de Puntos Limpios y que sean conocidos y accesibles a la comunidad.

En tercer lugar, se nombran acciones de defensa de áreas que probablemente están amenazadas o en riesgo de contaminación, luego se nombra el apoyo al trabajo que realizan las ONG internacionales, seguido de participación en actividades de limpieza organizadas por grupos sociales o instituciones, le sigue la adherencia a campañas de sensibilización y la participación en grupos escolares o que promueven una valoración del medio ambiente y la naturaleza (como Scouts). Si bien se nombra la recolección de firmas no se especifica en qué tema o área concreta. Por último, las y los jóvenes universitarios declaran su afinidad, sus conocimientos o la realización de prácticas sustentables como huertos, autocultivo y estilo de vida vegano como parte de las acciones que realizan para proteger al medio ambiente.

Las y los jóvenes estudiantes universitarios encuestados canalizan su preocupación por el medio ambiente participando en diversas actividades que promueven su protección, aunque estas acciones representan una minoría dentro del grupo juvenil encuestado. Sin embargo, este tipo de participación sería característica de la juventud contemporánea, ya que da cuenta de nuevas instancias de expresión, con estructuras informales o temporales, que no implican necesariamente un distanciamiento con la política o una actitud de rechazo hacia el sistema democrático, sino, nuevas formas de concebir lo político y lo juvenil (Castillo, 2007, Camargo, 2009, Báez, 2010, Arias-Cardona \& Alvarado, 2015); estrategias políticas como una marcha contra una hidroeléctrica o el reciclaje constituyen un nuevo escenario para llevar a cabo acciones de protección ambiental y son vistas por las y los jóvenes como actividades que promueven la protección del medio ambiente. Además, en el caso de las marchas, al ser un grupo social con estatus y poder 
(universitarios) logran legitimar sus demandas frente a la sociedad y, así, influir en el debate público.

\section{Conclusiones}

En primer lugar, se concluye que las y los jóvenes universitarios encuestados tienen una percepción negativa sobre las condiciones del medio ambiente urbano de la ciudad de Santiago, ya que la mayoría considera que son malas o muy malas, lo cual coincide con las mediciones técnicas realizadas por la institucionalidad respectiva, esta percepción es similar en términos de sexo. A pesar de esta negativa percepción sobre las condiciones ambientales, un poco más de la mitad de las personas encuestadas considera que el medio ambiente es igual de importante que el desarrollo económico, pero también un alto porcentaje pone en primer lugar al medio ambiente por sobre el desarrollo económico.

Por otro lado, la mayoría de las y los jóvenes percibe que los espacios públicos urbanos -como plazas y parques- en los que comparten a diario con sus amistades o familias están en un estado regular (limpios, pero poco equipados y con escasas áreas verdes). En términos de nivel socioeconómico, los sectores más pobres o excluidos de la ciudad de Santiago tienen menos espacios públicos urbanos en condiciones óptimas (limpios, equipados y con áreas verdes), lo que evidencia la falta de políticas públicas de desarrollo urbano integral y confirma lo hallado en otras investigaciones. Una abrumante mayoría de las y los jóvenes universitarios considera que la contaminación del medio ambiente urbano de Santiago más importante es la del aire y sus principales productores serían las industrias y los vehículos. Esta negativa percepción coincide con las mediciones técnicas de la institucionalidad respectiva, aunque difiere en las fuentes contaminantes: además de las mencionadas, se añade la calefacción residencial.

En segundo lugar, se concluye que las y los jóvenes universitarios encuestados tienen una actitud altamente positiva hacia el medio ambiente, ya que una aplastante mayoría señaló que los problemas ambientales le preocupan mucho. Los datos muestran que la gran mayoría de las y los jóvenes universitarios prefiere la educación ambiental como una solución a los problemas medioambientales, seguido de leyes más estrictas, incentivos y multas. Las mujeres se inclinan más por la educación, mientras que los hombres por leyes más estrictas y multas, coincidiendo con lo hallado en otras investigaciones. Un último aspecto en cuanto a la actitud, se refiere a que una abrumadora mayoría de las y los estudiantes universitarios encuestados considera que todos los actores sociales (gobierno, empresas, municipios y ciudadanía) son responsables del cuidado del medio ambiente y no solo uno; el área de Ciencias opta por actores privados como empresas y ciudadanía y las Ciencias Sociales, por el gobierno y el municipio, las mujeres se inclinan más por los actores locales (ciudadanía y municipio), mientras que los hombres, por actores nacionales (gobierno y empresas).

En tercer lugar, se concluye que las y los jóvenes universitarios tienen un alto conocimiento de los principales conceptos vinculados a la protección del medio ambiente, tales como reciclaje, contaminación atmosférica y del agua, cambio climático y deforestación, pero no ocurre lo mismo con temáticas emergentes como biodiversidad, eficiencia energética, servicios ecosistémicos, desertificación y especies invasoras, estos conocimientos han sido adquiridos principalmente a través de la educación secundaria y la televisión, pero escasamente en el espacio familiar.

Por último y a raíz de todos estos datos, se concluye que a pesar de tener una percepción negativa sobre las condiciones ambientales de Santiago, de una actitud altamente positiva hacia el medio ambiente y de manejar conocimientos específicos respecto a éste, una minoría de las y los jóvenes universitarios encuestados declara desarrollar comportamientos proambientales concretos para proteger el medio ambiente urbano y la naturaleza en general. Esta débil correlación entre una alta preocupación y una baja capacidad de acción en cuanto al medio ambiente es similar a la tendencia hallada en otras investigaciones. Los comportamientos proambientales mayormente realizados por las y los jóvenes encuestados son marchas 
callejeras o cicletadas masivas en rechazo a proyectos energéticos y acciones de reciclaje. Con todo, se evidencia la necesidad de implementar estrategias de educación ambiental en todos los programas curriculares universitarios, como un contenido transversal y permanente, más allá de la carrera o el tipo de universidad en que se estudie. Si bien las y los jóvenes encuestados demuestran una alta preocupación por el medio ambiente, tanto del entorno urbano local como de la naturaleza en general, y tienen un diagnóstico claro sobre los problemas que lo afectan, las actitudes positivas y los comportamientos proambientales son más bien escasos y ambiguos. Es importante considerar que las y los jóvenes universitarios constituyen un actor social con capacidad de incidencia en el debate público actual y con autoridad para tomar decisiones en el futuro. Estas dos dimensiones (incidencia y decisiones) eventualmente podrían impactar en mejorar las condiciones del medio ambiente urbano y la protección de la naturaleza en general, por ello, toda acción de sustentabilidad debería considerar su percepción, actitudes, conocimientos y comportamientos, puesto que son un actor social estratégico en el desarrollo de la ciudad y del país.

\section{Lista de referencias}

Aguayo, C. (2005). Análisis comparativo entre establecimientos educacionalescertificados $y$ no certificados ambientalmente en tres comunas de Santiago. Tesis, Departamento de Ingeniería Forestal, Universidad de Chile, Chile. Recuperado de: http://www. tesis.uchile.cl/tesis/uchile/2005/aguayo_c/ sources/aguayo_c.pdf.

Alea, A. (2006). Diagnóstico y potenciación de la educación ambiental en jóvenes universitarios. Odiseo, 3 (6), pp. 1-29.

Álvarez, P. \& Vega, P. (2009). Actitudes ambientales y conductas sostenibles. Implicaciones para la educación ambiental. Revista de Psicodidáctica, 14 (2), pp. 245260.

Arias-Cardona, A. M. \& Alvarado, S. V. (2015). Jóvenes y política: De la participación formal a la movilización informal. Revista
Latinoamericana de Ciencias Sociales, Niñez y Juventud, 13 (2), pp. 581-594. Doi :10.11600/1692715x.1322241014.

Avendaño, O. (2014). Fracturas y representación política en el movimiento estudiantil. Chile 2011. Última década, 41, pp. 4168. Doi: $\quad / / d x . d o i . o r g / 10.4067 / S 0718-$ 22362014000200003.

Báez, G. (2010). La participación protagónica estudiantil en el proceso de extensión universitaria. Revista Latinoamericana de Ciencias Sociales, Niñez y Juventud, 8 (1), pp. 347-362.

Barazarte-Castro, R., Neaman, A., VallejoReyes, F. \& García-Elizalde, P. (2014). El conocimiento ambiental y el comportamiento proambiental de los estudiantes de la Enseñanza media, en la Región de Valparaíso (Chile). Revista de Educación, 364, pp. 12-34. Doi: 10.4438/1988-592X-RE-2014-364-255.

Calvo-Cruz, X. (2013). La educación ambiental de la niñez costarricense en la edad escolar: Responsabilidad compartida por el currículo oficial del Ministerio de Educación Pública y el hogar. Biocenosis, 27 (1-2), pp. 14-20.

Camargo, M. (2009). La perspectiva de derechos en programas universitarios que forman para la Educación Inicial. Revista Latinoamericana de Ciencias Sociales, Niñez y Juventud, 7 (2), pp. 621-636.

Capp (2013). Informe País: Estado del Medio Ambiente en Chile 2012. Santiago de Chile: Centro de Análisis de Políticas Públicas, Instituto de Asuntos Públicos, Universidad de Chile.

Castillo, J. (2007). La configuración de las ciudadanías en estudiantes universitarios y universitarias de pregrado en Manizales, Colombia. Revista Latinoamericana de Ciencias Sociales, Niñez y Juventud, 5 (2), pp. 755-809.

Castro, A. \& Saavedra, E. (2013). Los jóvenes universitarios y las contradicciones en el ejercicio de ciudadanía, Katharsis, 16, pp. 293-310.

Chen, J. (2003). Across the Apocalypse on Horseback: Imperfect Legal Responses to Biodiversity Loss. The Jurisdynamics of 
Environmental Protection: Change and the Pragmatic. Washington: Environmental Law Institute.

De Mattos, C. (2002). Mercado metropolitano de trabajo y desigualdades sociales en el Gran Santiago: ¿Una ciudad dual? Eure, 28 (85), pp. 51-70. Doi: http://dx.doi. org/10.4067/S0250-71612002008500004.

Duerden, M. D. \& Witt, P. A. (2010). The impact of direct and indirect experiences on the development of environmental knowledge, attitudes, and behavior. Journal of Environmental Psychology, 30 (4), pp. 379392. Doi: 10.1016/j.jenvp.2010.03.007.

Félonneau, M. L. \& Becker, M. (2008). Proenvironmental attitudes and behavior: Revealing perceived social desirability. Revue Internationale de Psychologie Sociale, 21 (4), pp. 25-53.

Hess, S. \& Waló, W. (2001). Preocupación ambiental, conocimiento y uso de los puntos limpios en estudiantes universitarios. Medio Ambiente y Comportamiento Humano, 2 (2), pp. 39-56.

Heyl, M., Moyano-Díaz, E. \& Cifuentes, L. (2013). Environmental attitudes and behaviors of college students: A case study conducted at a Chilean university. Revista Latinoamericana de Psicología, 45 (3), pp. 487-500. Doi: http://dx.doi.org/10.14349/ rlp.v45i3.1489.

Holahan, C. (2006). Psicología Ambiental: Un Enfoque General. México, D. F.: Limusa.

Injuv (2013). 7ma Encuesta Nacional de Juventud 2012. Santiago de Chile: Imprenta Maval Ltda. Recuperado de: http://www. injuv.gob.cl/portal/wp-content/files_mf/ septimaencuestanacionaljuventud2.pdf.

Leal-Arancibia, F. \& Negron-Oyarzo, M. (2012). Tensiones socioambientales y rol de los medios regionales de comunicación en la formación de debate público: Dos casos emblemáticos para la institucionalidad ambiental chilena (Celco y Barrancones). Revista Austral de Ciencias Sociales, 22, pp. 25-42. Doi:10.4206/rev.austral.cienc. soc.2012.n22-02.

Le Breton, D. (1999). Las pasiones ordinarias. Antropología de las emociones. Buenos Aires: Nueva Visión.
Le Breton, D. (2013). Por una antropología de las emociones. Revista Latinoamericana de Estudios sobre Cuerpos, Emociones y Sociedad, 10 (4), pp. 69-79.

MEA (2005). Ecosystems and Human Wellbeing: Synthesis. Washington, D. C.: World Resources Institute.

Meinhold, J. L. \& Malkus, A. J. (2005). Adolescent environmental behaviors: Can knowledge, attitudes, and selfefficacy make a difference? Environment and Behavior, 37 (4), pp. 511-532. Doi: 10.1177/0013916504269665.

Ministerio del Medio Ambiente (2010). Guía para una educación ambiental localPedal. Santiago de Chile: Ministerio de Medio Ambiente-Gobierno de Chile \& Agencia de Cooperación Internacional del Japón-Chile. Recuperado de: http://www. mma.gob.cl/educacionambiental/1319/ articles-52858_Guia_Educacion_ Ambiental_Local_PEDAL.pdf

Ministerio del Medio Ambiente (2011). Informe del estado del medio ambiente: Ministerio del Medio Ambiente. Santiago de Chile: Ministerio del Medio Ambiente. Recuperado de: http://www.mma.gob. cl/1304/w3-article-52016.html.

Ministerio del Medio Ambiente (2013). Primer Reporte del Estado del Medio Ambiente. Santiago de Chile: Ministerio del Medio Ambiente. Recuperado de: http://www. mma.gob.cl/1304/w3-article-56613.html

Ministerio del Medio Ambiente (2014). Informe Primera Encuesta Nacional de Medio Ambiente. Santiago de Chile: Ministerio del Medio Ambiente. Recuperado de: http://portal.mma.gob.cl/wp-content/ uploads/2015/03/Informe-PrimeraEncuesta-Nacional-de-Medio-Ambiente. pdf

Ministerio del Medio Ambiente (2015). Ministerio del Medio Ambiente propone restricción para vehículos con sello verde y la prohibición del uso de leña en la Región Metropolitana. Recuperado de: http://portal.mma.gob.cl/ministerio-delmedio-ambiente-propone-restriccion-paracataliticos-y-la-prohibicion-del-uso-delena-en-la-region-metropolitana/ 
Moyano-Díaz, E., Encina, Y. \& Vicente, D. (2007). Evaluación del Sistema Nacional de Certificación Ambiental de Establecimientos Educacionales (Sncae) en Chile: operatoria e impacto. Psicologia para América Latina, 10, pp. 1-10. Recuperado de: http://pepsic. bvsalud.org/scielo.php?pid=S1870350X2007000200015\&script=sci_arttext

Muñoz-Pedreros, A. (2014). Environmental education in Chile: A pending task. Ambiente \& Sociedade, 17, pp. 177-198. Doi: $\quad$ http://dx.doi.org/10.1590/S1414$753 X 2014000300011$.

Novo, M. (2009). La educación ambiental, una genuina educación para el desarrollo sostenible. Revista de Educación, (número extraordinario), pp. 195-217.

Oliver-Trobat, M. F., Castells-Valdivielso, M., Casero-Martínez, A. \& Morey-López, M. (2005). Actitudes y percepción del medio ambiente en la juventud española. Madrid: Ministerio de Medio Ambiente.

Parker-Gumucio, C. \& Muñoz-Rau, J. (2012). Elites universitarias y cambio climático. Ambiente \& Sociedade, 25 (2), pp. 195218. Doi: http://dx.doi.org/10.1590/S1414$753 X 2012000200011$.

Parra, O. (2002). Actuales falencias en el aporte de la universidad al tema ambiental y desarrollo sustentable. Revista Ambiente y Desarrollo, 18 (2-3), pp. 208-213.

Reyes, S. \& Figueroa, I. (2010). Distribución, superficie y accesibilidad de las áreas verdes en Santiago de Chile, Eure, 36 (109), pp. 89-110. Doi:10.4067/S025071612010000300004.

Reynolds, J. F., Smith, D. M. S., Lambin, E. F., Turner, B. L., Mortimore, M., Batterbury ... Walker, B. (2007). Global Desertification: Building a Science for Dryland Development. Science, 316 (5826), pp. 847-851. Doi: http://dx.doi. org/10.1126/science.1131634.

Rojas, H. M. (2009). Formar investigadores e investigadoras en la universidad: Optimismo e indiferencia juvenil en temas científicos. Revista Latinoamericana de Ciencias Sociales, Niñez y Juventud, 7 (2), pp. 1595-1618.
Sala, O. E., Chapin, F. S., Armesto, J. J., Berlow, E., Bloomfield, J., Dirzo (2000). Global biodiversity scenarios for the year 2100. Science, 287 (5459), pp. 17701774. Doi: http://dx.doi.org/10.1126/ science.287.5459.1770.

Sevillano, V. \& Aragonés, J. (2009). Percepción social de la conducta de los españoles en materia medioambiental. Revista Española de Investigaciones Sociológicas, 126, pp. 127-149.

Stern, P. C. (2000). Toward a coherent theory of environmentally significant behavior. Journal of Social Issues, 56 (3), pp. 523530.

Taylor, S. \& Todd, P. (1995). An Integrated Model of Waste Management Behavior: A Test of Household Recycling and Composting Intentions. Environment and Behavior, 27 (5), pp. 603-630. Doi: http:// dx.doi.org/10.1177/0013916595275001.

Universidad Andrés Bello (2013). IV Encuesta de Percepción y Actitudes hacia el Medio Ambiente. Santiago de Chile: Universidad Andrés Bello-Centro de Investigación para la Sustentabilidad y Estudios Cuantitativos. Recuperado de:

http://ambiental.unab.cl/2013/04/34-delas-personas-declara-reciclar-en-chile/

Valenzuela, L., Justiniano, C., Araos, C., Katz, C., Andrade, M., Gática ... Torrent, C. (2010). Sustentabilidd en espacios colectivos de barrios vulnerables: Lineamientos para una política de espacios públicos, directrices de gestión, diseño y mantenimiento. Cuaderno de Investigación Urbanística, 68, pp. 83-104. 